\title{
Modulatory effect of PAR on PARP1-YB-1 interactions
}

K. Naumenko, 2*, E. Alemasova ${ }^{2}$, O. Lavrik ${ }^{1,2}$

${ }^{1}$ Novosibirsk State University, Novosibirsk, Russia

${ }^{2}$ Institute of Chemical Biology and Fundamental Medicine SB RAS, Russia

*e-mail: k-naumenko@mail.ru

Key words: Y-box binding protein 1 (YB-1), base excision repair (BER), poly(ADPribose)polymerase 1 (PARP1), poly(ADP-ribose) (PAR)

Motivation and Aim: Y-box-binding protein 1 (YB-1) is a multifunctional protein involved in a lot of cellular processes. YB-1 is a DNA- and RNA-binding protein. By binding to nucleic acids, YB-1 is engaged in many DNA- and mRNA-dependent processes such as DNA replication and repair, transcription and mRNA translation. Currently, YB-1 is considered as one of noncanonical proteins of base excision repair (BER). YB-1 has an increased affinity to damaged DNA and interacts in vivo and in vitro with several BER proteins, regulating their activities. Recently, it was shown that YB-1 also interacts with poly(ADP-ribose)polymerase 1 (PARP1), the key regulator of BER. PARP1 binding to damaged DNA results in its activation followed by synthesis of nucleic acid-like polymer of ADP-ribose (PAR) originating from $\mathrm{NAD}^{+}$. As a protein post-translational modification, PAR performs numerous functions in the regulation of BER. The main target of PARylation is PARP1 itself. It was found that PARP1 modify YB-1 with PAR, and YB-1 is able to stimulate the activity of PARP1. Interestingly, YB-1 also interacts non-covalently with PAR. The aim of present research was to investigate potential role of PAR on PARP1-YB-1 interactions.

Methods: Gel-mobility shift analysis, gel electrophoresis

Results and Conclusion: In the present work we explored the influence of purified PAR on YB-1-PARP1 interplay during PARylation. Our data demonstrate that PAR in low concentration may promote interactions of YB-1 and PARP1, resulting in increased modification of both proteins.

Conversely, YB-1 modification level and PARP1 activity are decreased by excess PAR due to disconnection of functional coupling of YB-1 and PARP1.To conclude, we found that the interaction of PARP1 and regulatory protein YB1 may be modulated by PAR during PARylation process.

Acknowledgements: This work was supported by grant from RSF (No. 14-24-00038). 